The AstrophysiCAL Journal, 334:L77-L80, 1988 November 15

(C) 1988. The American Astronomical Society. All rights reserved. Printed in U.S.A

\title{
EVIDENCE FOR ANOMALOUS COSMIC-RAY HYDROGEN
}

\author{
E. R. Christian, A. C. Cummings, and E. C. Stone \\ California Institute of Technology \\ Received 1988 August 8 ; accepted 1988 August 18
}

\begin{abstract}
We present evidence for the emergence of a measurable anomalous cosmic-ray hydrogen component in 1987 which may account for $20 \%-40 \%$ of the total hydrogen flux at $60 \mathrm{MeV}$. Comparing this flux with that of anomalous cosmic-ray helium, we estimate that the $\mathrm{H} \mathrm{I} / \mathrm{He} \mathrm{I}$ ratio in the very local interstellar medium is $\sim 4$, consistent with determinations from solar ultraviolet backscatter measurements.

Subject headings: cosmic rays: abundances - cosmic rays: general - interstellar: abundances
\end{abstract}

\section{INTRODUCTION}

Anomalous cosmic rays (ACR) are believed to be interstellar neutrals (Fisk, Kozlovsky, and Ramaty 1974) which are ionized near the Sun, convected outward by the solar wind, and accelerated at the termination shock (Pesses, Jokipii, and Eichler 1981; Jokipii 1986; Potgieter and Moraal 1988). He, N, $\mathrm{O}$, and Ne have long been known to have an ACR component (Garcia-Munoz, Mason, and Simpson 1973; McDonald et al. 1974; Hovestadt et al. 1973), while the anomalous cosmic-ray components of argon and carbon are smaller and have only recently been reported (Cummings and Stone 1987).

It has been suggested that hydrogen, which is the most abundant neutral in the interstellar medium and which is easily ionized by photoionization or charge exchange with the solar wind, should also have an ACR component (see, e.g., Fisk 1986), although no observations have been reported. However, as pointed out by Fisk (1986), the likely peak energy and shape of an anomalous cosmic-ray hydrogen spectrum may be very similar to the modulated galactic cosmic-ray (GCR) hydrogen spectrum, thus making it difficult to distinguish between the two. In this Letter we examine the energy spectra of hydrogen observed near the time of maximum fluxes in 1985 to 1987 from instruments on the Voyager $1(V I)$ and Voyager $2(V 2)$ spacecraft and find changes which represent the first evidence for the existence of anomalous cosmic-ray hydrogen.

\section{DATA ANALYSIS}

All of the spectra in this analysis were obtained in the outer heliosphere ( $\geq 18$ AU) with the Cosmic Ray Subsystem (CRS) on the two Voyager spacecraft (Stone et al. 1977). Hydrogen energy spectra for the two time periods 1985/261-365 and 1987/209-313 are shown for Voyager 1 and Voyager 2 in Figure 1. The spectra have been divided by energy to accentuate the differences in spectral shape. The curves which have been drawn through the two 1985 spectra are also scaled up in flux to match the 1987 spectra at the highest energy and illustrate the change in spectral shape that has occurred between the two periods. The high-energy points $(\geq 150 \mathrm{MeV})$ of these spectra are from the analysis of particles which fully penetrate the CRS high-energy telescopes (HETs) and have been corrected for a background which is less than $10 \%$ of the observed flux. Since the discussion in this Letter depends only on the relative differences between similar spectra, omitting this correction would result in only small quantitative differences in the results. The 10-72 MeV fluxes are from the analysis of protons that stop in the telescopes and require no correction.

\section{DISCUSSION}

The major difference between the two time periods in the spectra of Figure 1 is the increase in flux resulting from a decrease in the solar modulation level between 1985 and 1987. Both $V 1$ and $V 2$ spectra show a change in shape, which is more pronounced in $V 2$. As discussed below, we believe that this change is evidence of the appearance of an anomalous hydrogen component during the period of minimum solar modulation.

To obtain an upper estimate for a presumed anomalous hydrogen flux in the 1987 spectra of Voyager 1 and Voyager 2 , we subtract estimates of the GCR flux which were determined by normalizing the 1985 spectra to the 1987 spectra at the highest energy (363 MeV), as shown by the dashed lines in Figure 1. This approach might be expected to underestimate the anomalous cosmic-ray hydrogen flux by $10 \%-20 \%$, because the 1985 spectra should include an anomalous component which is about $10 \%$ to $20 \%$ of that in 1987 , as is the case for ACR helium as shown in Figure 2. A further underestimate might be expected because there is likely a small anomalous contribution to the 1987 flux at the normalizing energy of $363 \mathrm{MeV}$. However, this approach will overestimate the anomalous flux to the extent that some of the change in spectral shape between 1985 and 1987 is due to changes in the spectrum of the modulated GCR flux.

Although modulation theory is not understood well enough to allow accurate calculations of spectra, we can use other observational evidence to estimate modulation induced changes in the spectra. For example, the peaks in the galactic cosmic-ray helium spectra in Figure 2 shifted downward by $21 \%$ between 1985 and 1987 on both Voyager 1 and Voyager 2. An energy shift of $21 \%$ for the $V 1$ hydrogen spectra also results from a least-squares fit of the 1985 spectrum to that of 1987 , assuming there is negligible anomalous flux in the $V 1$ spectra. If it is assumed that there was a similar energy shift in the $V 2$ GCR hydrogen spectra, then an upper estimate of the V2 1987 GCR hydrogen spectrum can be made by shifting the V2 1985 spectrum downward $21 \%$ in energy and normalizing it in flux to the $V 21987$ spectrum at the highest energy measured. Subtracting this shifted spectrum from the observed $V 2$ 1987 hydrogen spectrum results in a lower estimate of the ACR 


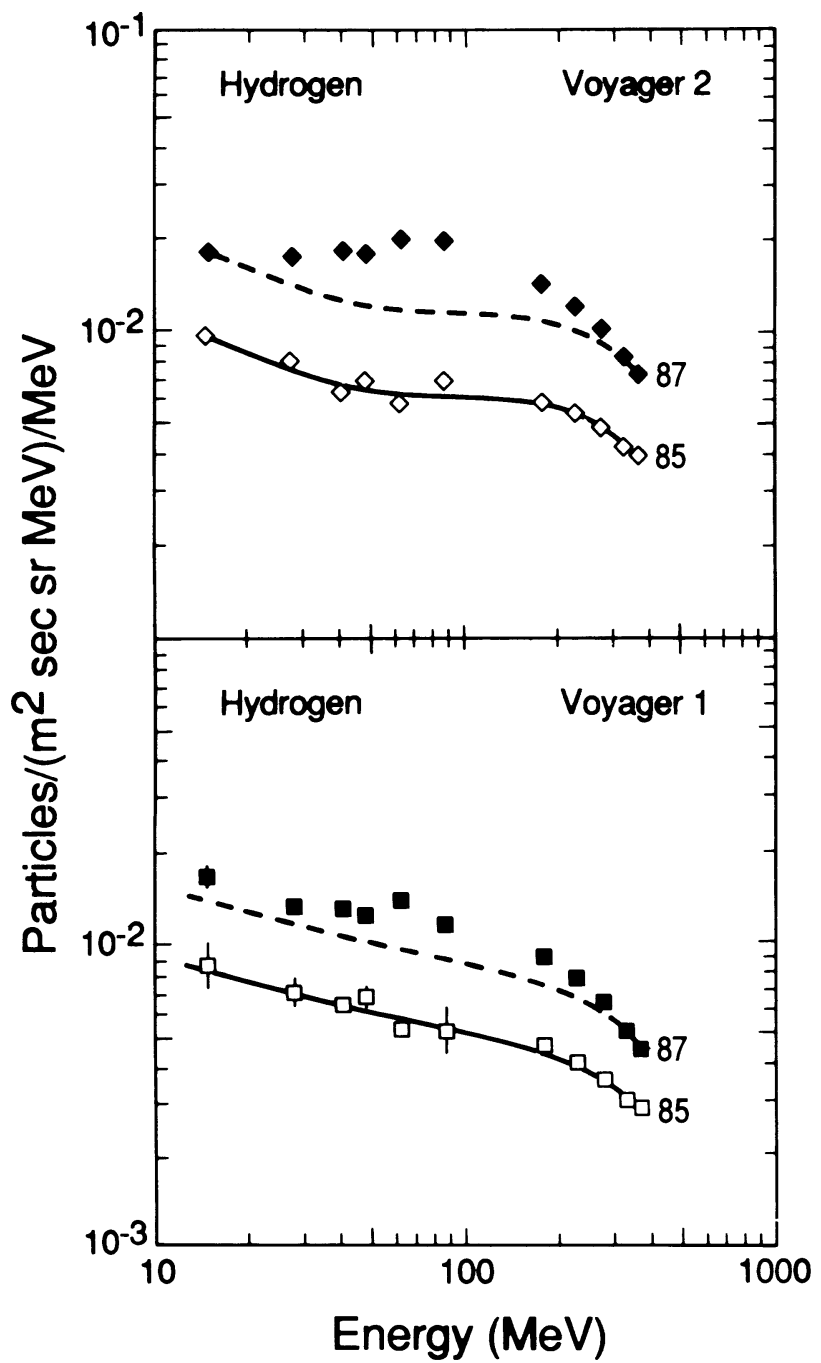

FIG. 1.-Observed hydrogen energy spectra for the time periods $1985 / 261$ 365 and 1987/209-313. The spectra have been divided by energy to emphasize changes. The dashed curves for both Voyagers are the same shape as the solid curves drawn through the respective 1985 spectra except shifted in flux to match the 1987 spectra at the highest energy point $(363 \mathrm{MeV})$. The error bars include only statistical uncertainties.

hydrogen flux in Voyager 2. This approach is expected to underestimate the $V 2$ anomalous cosmic-ray hydrogen flux in 1987 because it assumes that there was no anomalous contribution to the 1985 spectra and that there was negligible anomalous flux in the VI 1987 spectrum.

Figure 3 shows both this lower estimate and the previously derived upper estimates for the anomalous hydrogen spectra, as well as the observed 1987 energy spectra. For Voyager 2 we estimate that the ACR hydrogen component is $\sim 20 \%-40 \%$ of the total hydrogen flux at $\sim 60 \mathrm{MeV}$. The shape of the upper estimates of the Voyager 1 and Voyager 2 spectra are essentially the same, with the Voyager 1 fluxes a factor of 2 lower as expected from the large negative latitudinal gradients observed for the other ACR components (Cummings, Stone, and Webber 1987).

In order to further investigate the possibility that the spectral changes in Figure 1 might be due to a peculiar modulation effect rather than to anomalous cosmic-ray hydrogen, we have examined the spectral shapes of other galactic cosmic-ray nuclei. Because modulation depends on the magnetic rigidity of the particle and hydrogen has only half the mass per charge of heavier nuclei, modulation related features will appear at higher energies in the hydrogen spectrum than in the others. Thus, the maximum GCR hydrogen flux in Figure 3 occurs at $\sim 280 \mathrm{MeV}$, while the maximum GCR helium flux occurs at $\sim 210 \mathrm{MeV}$ per nucleon. As a result, if the excess hydrogen flux at $100 \mathrm{MeV}$ was due to modulation, a similar excess would be expected at $\sim 75 \mathrm{MeV} /$ nucleon for heavier galactic cosmic-ray nuclei. Carbon is the only sufficiently abundant heavier nucleus that is relatively free of contamination by the anomalous component at this energy (Cummings and Stone 1987).

Figure 4 shows the carbon flux-divided-by-energy spectra for both Voyagers in the time periods 1985/261-1986/105 and 1987/105-313. The time periods are double the length of those for hydrogen to reduce the statistical uncertainty. Averaging over these longer time periods for hydrogen would dilute the observed spectral shape change seen in hydrogen by only $10 \%-15 \%$. As in Figure 1, the curves from the 1985/1986 spectra are also scaled up in energy to match the 1987 spectra at the highest energy. Except for an increase in the ACR com-

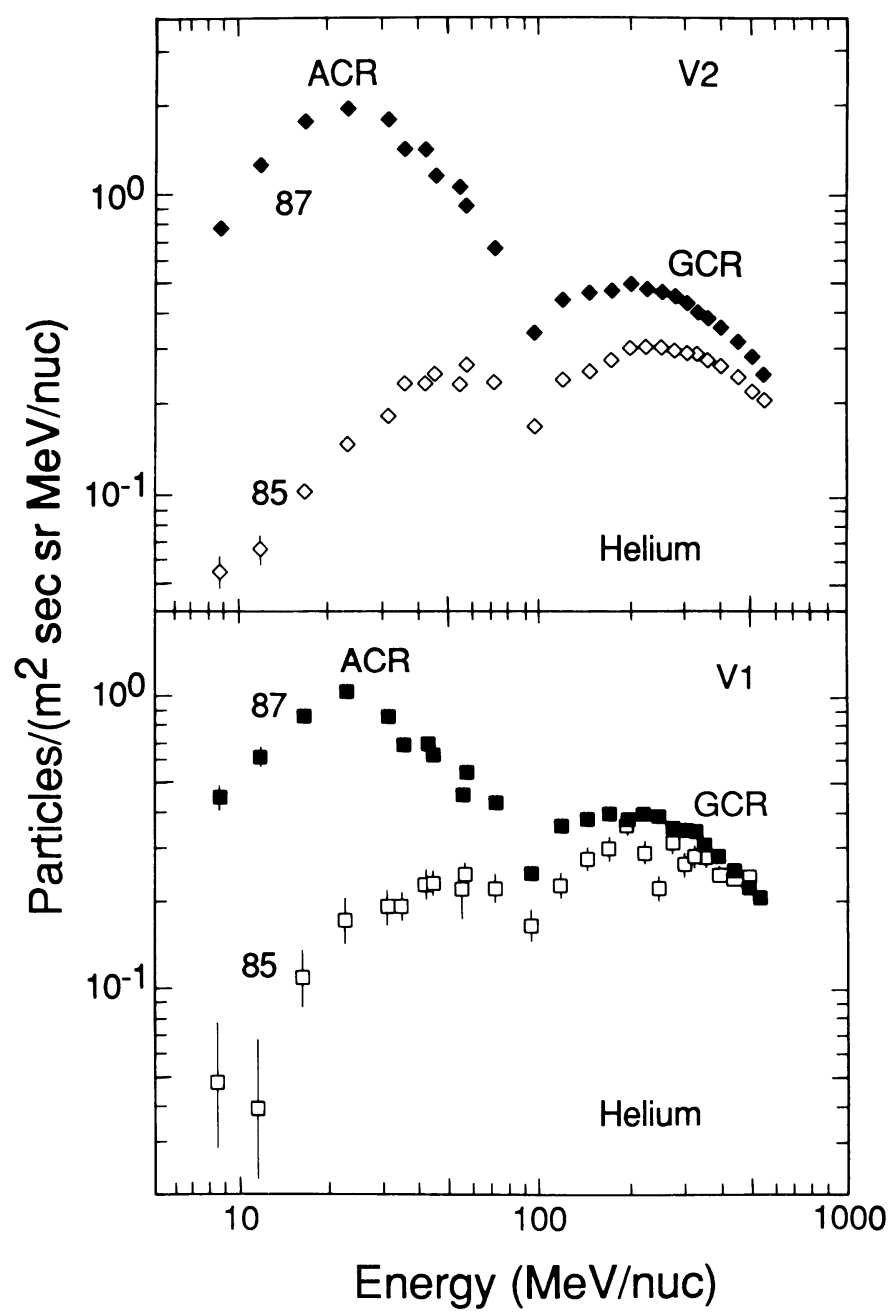

Fig. 2.-Observed helium energy spectra for the time periods $1985 / 261-$ 365 and 1987/209-313. Below $\sim 100 \mathrm{MeV}$ per nucleon, the spectra are predominantly due to anomalous cosmic rays (ACR), and above $100 \mathrm{MeV}$ per nucleon the spectra are dominated by galactic cosmic rays (GCR). The error bars include only statistical uncertainties. 


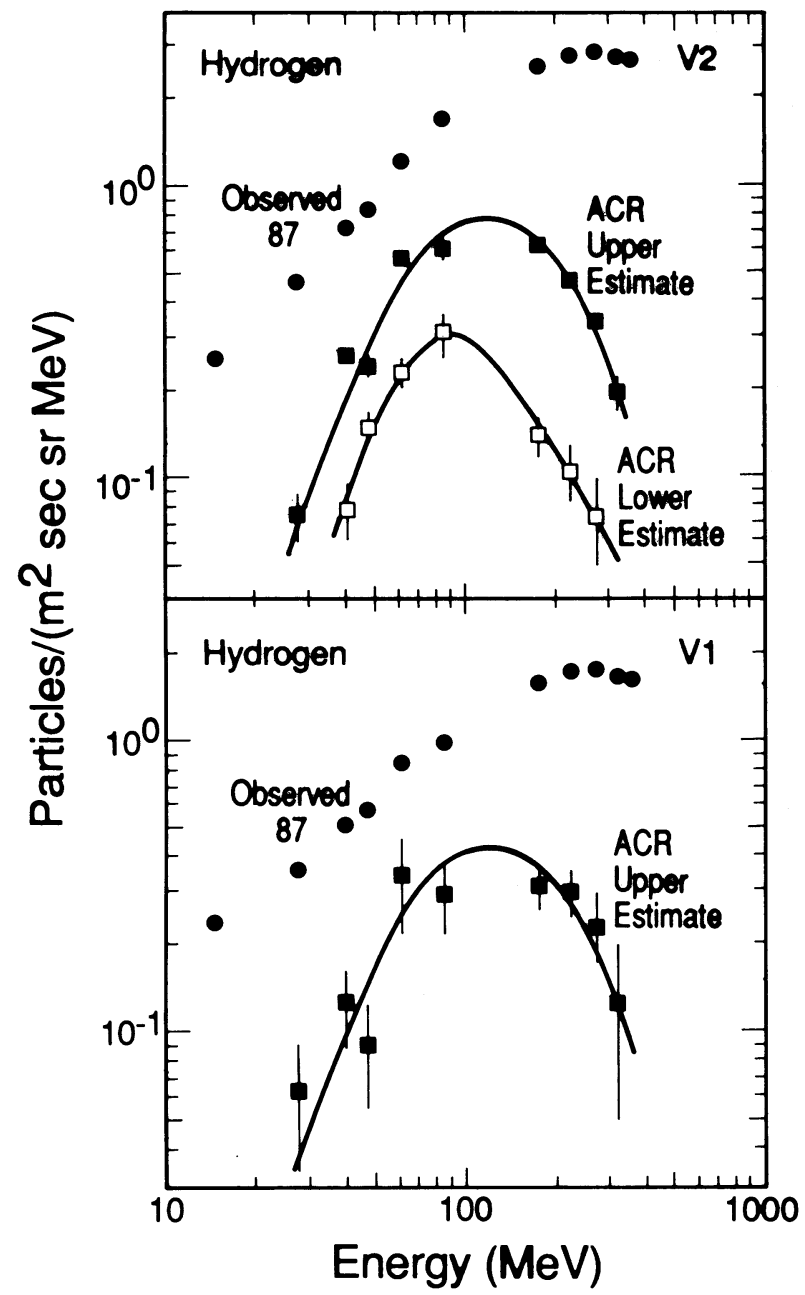

Fig. 3.-Estimates of anomalous hydrogen energy spectra during the time period 1987/209-313. The solid curves for the upper and lower estimates in $V 2$ are to guide the eye and are not fits to the data. The solid curve for $V l$ is the curve for the upper estimate of $V 2$ shifted down by a factor of 2 in flux. Also included are the observed hydrogen energy spectra for both Voyagers during this time period. The error bars include only statistical uncertainties.

ponent at low energies (Cummings and Stone 1988), there is no apparent change in shape at $\sim 75 \mathrm{MeV}$ per nucleon between 1985 and 1987. The comparison of Figures 1 and 4 thus suggests that the excess flux in Voyager 2 hydrogen is not solely a result of the modulation of a purely galactic cosmic-ray spectrum.

Assuming that the excess flux is evidence for an anomalous component, we can then use the mean of the upper and lower estimates in Figure 3 as a measure of the anomalous cosmicray hydrogen flux at Voyager 2, with an uncertainty that includes both the lower and the upper estimate. When the peak anomalous hydrogen flux of Voyager 2 is compared with the peak $V 2$ anomalous helium flux in Figure 2, we obtain an observed $\mathrm{H} / \mathrm{He}$ flux ratio of $0.20 \pm 0.08$. As discussed in Cummings and Stone (1987), the relative abundances of neutral atoms in the very local interstellar medium (VLISM) can be estimated from the relative ACR peak fluxes. This requires that the observed ACR ratio be corrected for differences in the fraction of $\mathrm{H} \mathrm{I}$ and $\mathrm{He}_{\mathrm{I}}$ ionized in the heliosphere and for differences in the acceleration and subsequent modulation of the resulting ions arising from their differing mass per unit charge.

In order to correct for the relative ionization fractions, we have used the values for ionization rates determined from the solar ultraviolet backscatter experiment on Prognoz 5 and Prognoz 6, which are $3 \pm 1 \times 10^{-7} \mathrm{~s}^{-1}$ (Bertaux et al. 1985) and $1.25 \pm 0.23 \times 10^{-7} \mathrm{~s}^{-1}$ (Dalaudier et al. 1984) for $\mathrm{H}$ and $\mathrm{He}$, respectively. The Prognoz 5 and Prognoz 6 measurements were made mainly in 1977 when the solar activity, as indicated by the $10.7 \mathrm{~cm}$ solar flux (National Geophysical Data Center 1988), was similar to that during the 1987 time period of the Voyager measurements. When combined with a simple ionization model similar to that outlined by Axford (1972), these rates give ionization efficiencies of $0.073 \pm 0.021$ for hydrogen and $0.036 \pm 0.006$ for helium. Thus the correction factor for the relative ionization efficiencies, $F_{\text {ion }}$, is $0.49 \pm 0.16$.

Differences in the acceleration and modulation of the ions is more difficult to estimate accurately because of incomplete

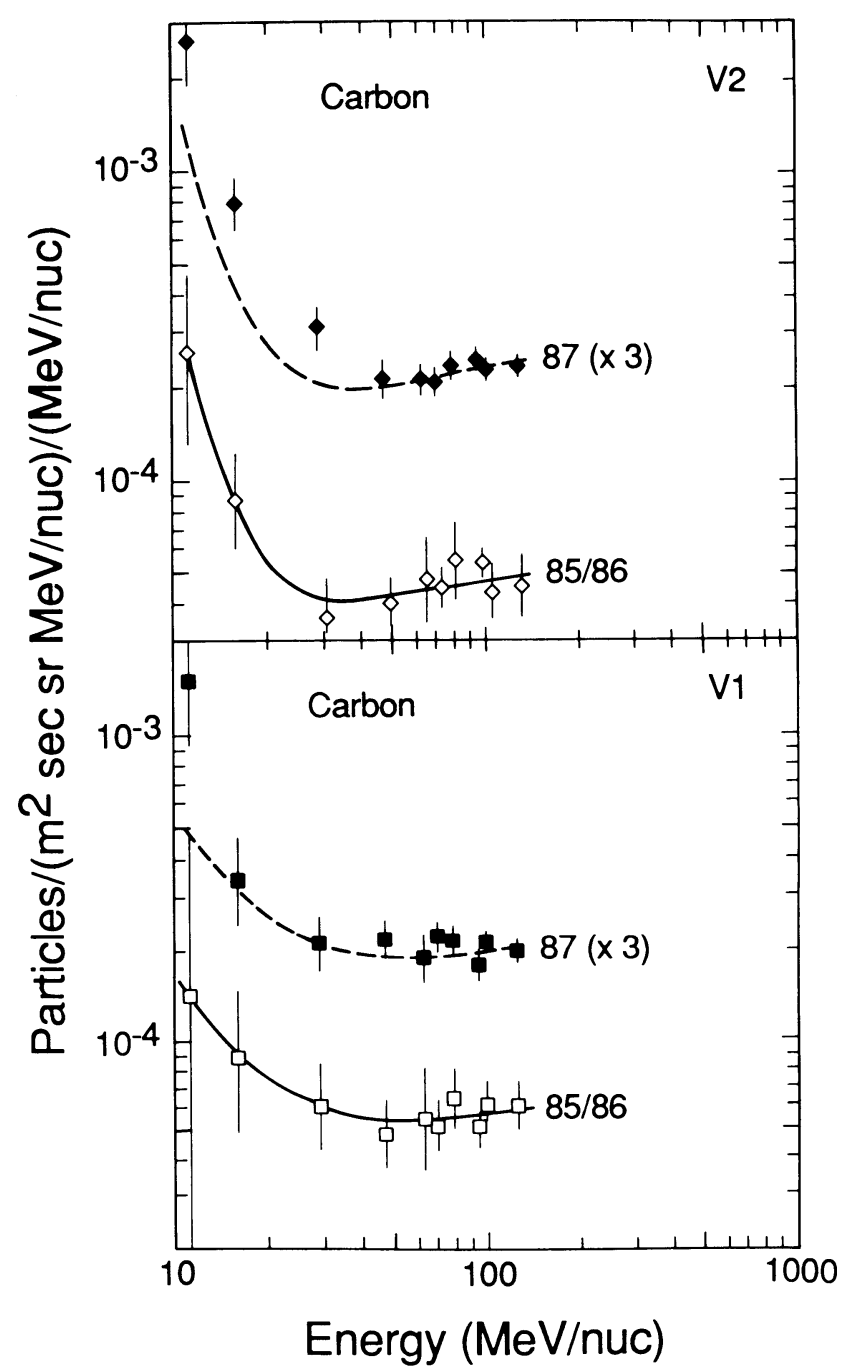

Fig. 4. - Observed carbon energy spectra for the time periods $1985 / 261$ $1986 / 105$ and 1987/105-313, with the 1987 spectra for both Voyagers multiplied by 3 . The spectra have been divided by energy per nucleon to emphasize changes. The dashed curves for both Voyagers are the same shape as the solid curves drawn through the respective $1985 / 1986$ spectra except shifted in flux to match the 1987 spectra at the highest energy point (125 MeV per nucleon). The error bars include only statistical uncertainties. 
theoretical understanding. Cummings and Stone (1987)

's' examine acceleration correction factors for one acceleration

, model, finding that if equal abundances of hydrogen and

Iol helium ions are injected at the same velocity (e.g., the solar

$I-I$ wind velocity), the peak fluxes of accelerated and modulated helium would be $\sim 37$ times that of hydrogen. Although the uncertainty in this value is difficult to assess, it is nevertheless illustrative to use this as the acceleration correction factor, $F_{\text {acc }}$. This results in a $\mathrm{H} \mathrm{I} / \mathrm{He} \mathrm{I}$ abundance of $4 \pm 2$ in the VLISM, as given by $F_{\text {ion }} \times F_{\text {acc }} \times(\mathrm{H} / \mathrm{He})_{\mathrm{ACR}}$, where the indicated uncertainty excludes the unknown uncertainty in $F_{\text {acc }}$ and any variation in $F_{\text {ion }}$ due to changes in solar activity. This $\mathrm{H} \mathrm{I} / \mathrm{He}_{\mathrm{I}}$ ratio is comparable to the ratios of $6.5 \pm 3.1$ (Chassefière et al. 1986) and $7 \pm 3$ (Ajello et al. 1987) derived from solar ultraviolet backscatter results. Although there are modeling uncertainties in this estimate of $\mathrm{H} \mathrm{I} / \mathrm{He} \mathrm{I}$, the agreement with previous values suggests that with further theoretical work it may be possible to derive accurate relative abundances of neutrals in the VLISM from observations of the composition of the anomalous cosmic-ray component.

We thank F. B. McDonald and W. R. Webber for their helpful discussions and suggestions. We also appreciate the contributions of the other Voyager CRS team members at Caltech and the Goddard Space Flight Center. This work was supported in part by NASA under contract NAS 7-918 and grant NGR 05-002-160.

\section{REFERENCES}

Ajello, J. M., Stewart, A. I., Thomas, G. E., and Graps, A. 1987, Ap. J., 317, 964.

Axford, W. I. 1972, in Solar Wind, ed. C. P. Sonnett, P. J. Coleman, and J. M. Wilcox (NASA SP-308) (Washington: NASA), p. 609.

Bertaux, J. L., Lallement, R., Kurt, V. G., and Mironova, E. N. 1985, Astr. Ap., 150, 1 .

Chassefière, E., Bertaux, J. L., Lallement, R., and Kurt, V. G. 1986, Astr. Ap., $160,229$.

Cummings, A. C., and Stone, E. C. 1987, in Proc. 20th Internat. Cosmic Ray Conf. (Moscow), 3, 413 .

1988, in Proc. of Sixth Internat. Solar Wind Conf., ed. V. J. Pizzo, T. E.

Holzer, and D. G. Sime (Estes Park), 2, 599.

Cummings, A. C., Stone, E. C., and Webber, W. R. 1987, Geophys. Res. (Letters), 14, 174.

(Lett. 1987, Geophys. Res. (Letters), 14, 174.

Dalaudier, F., Bertaux, J. L., Kurt, V. G., and Mironova, E. N. 1984, Astr. Ap., 134, 171 .

Fisk, L. A. 1986, in The Sun and the Heliosphere in Three Dimensions, ed. R. G. Marsden (Dordrecht: Reidel), p. 401.

Fisk, L. A., Kozlovsky, B., and Ramaty, R. 1974, Ap. J. (Letters), 190, L35.

Garcia-Munoz, M., Mason, G. M., and Simpson, J. A. 1973, Ap. J. (Letters), 182, L81.

Hovestadt, D., Vollmer, O., Gloeckler, G., and Fan, C. Y. 1973, Phys. Rev. (Letters), 31, 650.

Jokipii, J. R. 1986, J. Geophys. Res., 91, 2929.

McDonald, F. B., Teegarden, B. J., Trainor, J. H., and Webber, W. R. 1974, Ap. J.(Letters), 187, L105.

National Geophysical Data Center. 1988, Ottawa $10.7 \mathrm{~cm}$ Solar Flux Diskette (Boulder: NOAA Environmental Data Information Service).

Pesses, M. E., Jokippi, J. R., and Eichler, D. 1981, Ap. J. (Letters), 246, L85.

Potgieter, M. S., and Moraal, H. 1988, A p. J., 330, 445.

Stone, E. C., Vogt, R. E., McDonald, F. B., Teegarden, B. J., Trainor, J. H., Jokipii, J. R., and Webber, W. R. 1977, Space Sci. Rev., 21, p. 355

E. R. Christian, A. C. Cummings, and E. C. Stone: 220-47 Downs Laboratory, California Institute of Technology, Pasadena, CA 91125 


\section{PLATE 22}

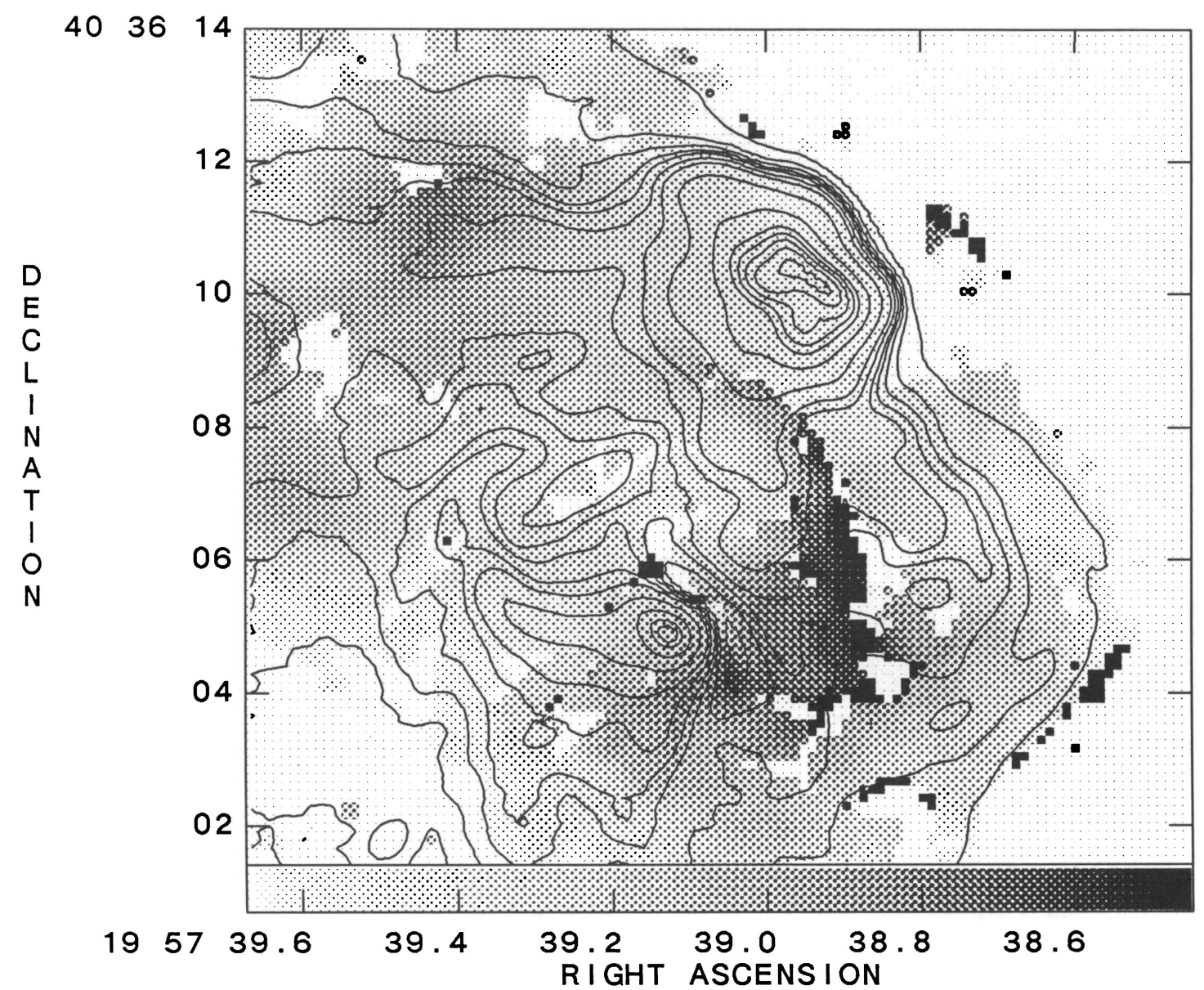

FIG. 1.-An image of the hot spots in the northwest lobe of Cygnus A. Contours are of total intensity at $4525 \mathrm{MHz}$, while the gray scale is the rotation measure (RM) distribution. The effective resolution (FWHM of the restoring beam) for the image is 0"4. The RM gray scale ranges from -200 to $1000 \mathrm{rad} \mathrm{m}^{-2}$. Hot spot B is the more compact hot spot to the south, while $A$ is the larger hot spot to the north. Notice the arc of large $R M \approx 3^{\prime \prime}$ west and north of hot spot $B$. This feature we interpret as the radio-quiet bow shock induced by the supersonic advance of the primary into the IGM.

CARILLI et al. (see 334, L22) 